\title{
Sliding Mode Control Design for Induction Motors: An Input-Output Approach
}

\author{
John Cortés-Romero ${ }^{1}$, Alberto Luviano-Juárez ${ }^{2}$ and \\ Hebertt Sira-Ramírez ${ }^{3}$ \\ ${ }^{1}$ Universidad Nacional de Colombia. Facultad de Ingeniería, Departamento de Ingeniería \\ Eléctrica y Electrónica. Carrera 30 No. 45-03 Bogotá \\ 1,2,3 Cinvestav IPN, Av. IPN No. 2508, Departamento de Ingeniería Eléctrica, Sección de \\ Mecatrónica \\ ${ }^{1}$ Colombia \\ ${ }^{2,3}$ México
}

\section{Introduction}

Three-phase induction motors have been widely used in a variety of industrial applications. Induction motors have been able to incrementally improve energy efficiency to satisfy the requirements of reliability and efficiency, Melfi et al. (2009). There are well known advantages of using induction motors over permanent magnet DC motors for position control tasks; thus, efforts aimed at improving or simplifying feedback controller design are well justified.

There exists a variety of control strategies that depend on difficult to measure motor parameters while their closed loop behavior is found to be sensitive to their variations. Even adaptive schemes tend to be sensitive to speed-estimation errors, yielding to a poor performance in the flux and torque estimation, especially during low-speed operation, Harnefors \& Hinkkanen (2008).

Generally speaking, the designed feedback control strategies have to exhibit a certain robustness level in order to guarantee an acceptable performance. It is possible to (on-line or off-line) obtain estimates of the motor parameters, Hasan \& Husain (2009); Toliyat et al. (2003), but some of them can be subject to variation when the system is undergoing actual operation. Frequent misbehavior is due to external and internal disturbances, such as generated heat, that significantly affect some of the system parameter values. An alternative to overcome this situation is to use robust feedback control techniques which take into account these variations as unknown disturbance inputs that need to be rejected. In this context, sliding mode techniques are a good alternative due to their disturbance rejection capability (see for instance, Utkin et al. (1999)).

In this chapter, we consider a two stage control scheme, the first one is devoted to the control of the rotor shaft position. This analog control is performed by means of the stator current inputs, in a configuration of an observer based control. The mathematical model of the rotor dynamics is a simplified model including additive, completely unknown, lumping nonlinearities and external disturbances whose effect is to be determined in an on-line fashion by means of linear observers. The gathered knowledge will be used in the appropriate canceling of the assumed perturbations themselves while reducing the underlying control problem to a simple linear feedback control task. The control scheme thus requires a rather reduced set of parameters to be implemented. 
The observation scheme for the modeled perturbation is based on an extension of the Generalized Proportional Integral (GPI) controller, Fliess, Marquez, Delaleau \& Sira-Ramírez (2002) to their dual counterpart: the GPI observer which corresponds to a class of extended Luenberger-like observers, Luviano-Juárez et al. (2010). Such observers were introduced in, Sira-Ramirez, Feliu-Batlle, Beltran-Carbajal \& Blanco-Ortega (2008) in the context of Sigma-Delta modulation observer tasks for the detection of obstacles in flexible robotics. Under reasonable assumptions, the observation technique consists in viewing the measured output of the plant as generated by an equivalent perturbed pure integration dynamics with an additive perturbation input lumping, in a single function, all the nonlinearities of the output dynamics. The linear GPI observer, is set to approximately estimate the states of the pure integration system as well as the evolution of the, state dependent, perturbation input. This observer allows one to approximately estimate, on the basis of the measured output, the states of the nonlinear system, as well as to closely estimate the unknown perturbation input. The proposed observation scheme allows one to solve, rather accurately, the disturbance estimation problem.

Here, these observers are used in connection with a robust controller design application within the context of high gain observation. This approach is prone to overshot effects and may be deemed sensitive to saturation input constraints, specially when used in a high gain oriented design scheme via the choice of large eigenvalues. Such a limitation is, in general, an important weakness in many practical situations. However, since our control scheme is based on a linear observer design that can undergo temporary saturations and smooth "clutchings" into the feedback loop, its effectiveness can be enhanced without affecting the controller structure and the overall performance. We show that the observer-based control, overcomes these adverse situations while enhancing the performance of the classical GPI based control scheme.

The linear part of the controller design is based on the Generalized Proportional Integral output feedback controller scheme established in terms of Module Theory.

In the second design stage, the designed current signals of the first stage are deemed as reference trajectories, and a discontinuous feedback control law for the input voltages is sought which tracks the reference trajectories. Since the electrical subsystem is faster than the mechanical, we propose a sliding mode control approach based on a class of filtered sliding surfaces which consist in regarding the traditional surface with the addition of a low pass filter, without affecting the relative degree condition of the sliding surface. The "chattering effect" related to the sliding mode application is eased by means of a first order low-pass filter as proposed in, Utkin et al. (1999).

GPI control has been established as an efficient linear control technique (See Fliess et al., Fliess, Marquez, Delaleau \& Sira-Ramírez (2002)); it has been shown, in, Sira-Ramírez \& Silva-Ortigoza (2006), to be intimately related to classical compensator networks design. The main limitation of this approach lies in the assumption that the available output signal coincides with the system's flat output (See Fliess et al.Fliess et al. (1995), and also Sira-Ramírez and Agrawal, Sira-Ramírez \& Agrawal (2004)) and, hence, the underlying system is, both, controllable and, also, observable from this special output. Nevertheless, this limitation is lifted for the case of the induction motor system.

The controller design is carried out with the philosophy of the classical field oriented controller scheme and implemented through a flux simulator, or reconstructor (see Chiasson, Chiasson (2005)). The methodology is tested and illustrated in an actual laboratory implementation of the induction motor plant in a position trajectory tracking task.

The rest of the chapter is presented as follows: Section 2 describes each of the methodologies to use along the chapter such as the sliding mode control method, the Generalized Proportional Integral control and the disturbance observer. The modeling of the motor and the problem formulation are given in Section 3, and the proposed methodologies are joined to solve the 
problem in Section 4. The results of the approach are obtained in an experimental framework, as depicted in Section 5. Finally some concluding remarks are given.

\section{Some preliminary aspects}

2.1 Sliding mode control using a proportional integral surface: Introductory example Consider the following first order system:

$$
\dot{y}=u+\xi(t)
$$

where $y$ is the output of the system, $\xi(t)$ can be interpreted as a disturbance input (which may be state dependent) and $u \in\{-W, W\}$ is a switched class input. We propose here to take as a sliding surface coordinate function the following expression in Laplace domain $s$ :

$$
\begin{gathered}
\sigma=-\frac{s+z}{s} e \\
e=y-y^{*}
\end{gathered}
$$

with $z>0$.

The switched control is defined as

$$
\begin{aligned}
u & =W \operatorname{sign}(\sigma), \\
W & >0
\end{aligned}
$$

We propose the following Lyapunov candidate function:

$$
V=\frac{1}{2} \sigma^{2}
$$

whose time derivative is $\dot{V}=\sigma \dot{\sigma}$. From (2)

$$
\dot{\sigma}=-\dot{e}-z e
$$

We have

$$
\begin{aligned}
\sigma \dot{\sigma} & =-\sigma \dot{e}-z e \sigma \\
& =-\sigma \dot{y}+\sigma \dot{y}^{*}-z e \sigma \\
& =-W|\sigma|-\sigma \xi(t)+\sigma \dot{y}^{*}-z e \sigma
\end{aligned}
$$

since the term $-\sigma \xi(t)+\sigma \dot{y}^{*}-z e \sigma$ does not depend on the input, by setting $W$ in such a way that we can ensure that $\dot{V}<0$, the sliding condition for $\sigma$ is achieved.

The classical interpretation of the output feedback controller suggests, immediately, the following discontinuous feedback control scheme:

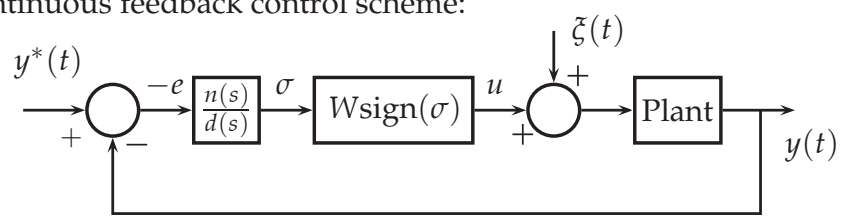

Fig. 1. GPI control scheme. 
where $n(s)=s+z$ regulates the dynamic behavior of the tracking error and $d(s)=s$ acts as a "filter" of the sliding surface.

The equivalent control is obtained from the invariance conditions:

$$
\sigma=\dot{\sigma}=0
$$

i.e,

$$
u_{e q}=\dot{y}^{*}-z e
$$

in other words, the proposed sliding surface has, in the equivalent control sense, the same behavior of the traditional proportional sliding surface of the form $\sigma_{1}=z e$. However, the closed loop behavior of the system with the smooth sliding surface, presents some advantages as shown in, Slotine \& Li (1991). Since this class of controls induce a "chattering effect", to reduce this phenomenon, we insert in the control law output a first order low-pass filter, which, in some cases, needs and auxiliary control loop (as shown in the integral sliding mode control design, Utkin et al. (1999)). In our case, the architecture of the control system based on two control loops and disturbance observers will act as the auxiliary control input.

\subsection{Generalized Proportional Integral Control}

GPI control, or Control based on Integral Reconstructors, Fliess \& Sira-Ramírez (2004), is a recent development in the literature on automatic control. Its main line of development rests within the finite dimensional linear systems case, with some extensions to linear delayed differential systems and to nonlinear systems (see Fliess et al., Fliess, Marquez, Delaleau \& Sira-Ramírez (2002), Fliess et al., Fliess, Marquez \& Mounier (2002) and Hernández and Sira-Ramírez, Hernández \& Sira-Ramírez (2003)).

The main idea of this control approach is the use of structural reconstruction of the state vector. This means that states of the system are obtained modulo the effect of unknown initial conditions as well as constant, ramp, parabolic, or, in general, polynomial, additive external perturbation inputs. The reconstructed states are computed solely on the basis of inputs and outputs. These state reconstructions may be used in a linear state feedback controller design, provided the feedback controller is complemented with a sufficient number of iterated output, or input, integral error compensation which structurally match the effects of the neglected perturbation inputs and initial states.

To clarify the idea behind GPI control, consider the following elementary example,

$$
\begin{aligned}
\ddot{y} & =u+\xi \\
y(0) & =y_{0} \\
\dot{y}(0) & =\dot{y}_{0}
\end{aligned}
$$

with $\xi$ being an unknown constant disturbance input. The control problem consists in obtaining an output feedback control law, $u$, that forces $y$ to track a desired reference trajectory, given by $y^{*}(t)$, in spite of the presence of the unknown disturbance signal and the unknown value of $\dot{y}(0)$.

Let $e_{y} \triangleq y-y^{*}(t)$ be the reference trajectory tracking error and let $u^{*}$ be a feed-forward input nominally given by $\ddot{y}^{*}(t)=u^{*}(t)$. The input error is defined as $e_{u} \triangleq u-u^{*}(t)=u-\ddot{y}^{*}(t)$. Integrating equation (7) we have, 


$$
\dot{y}=\int_{0}^{t} u(\tau) d \tau+\dot{y}(0)+\xi t
$$

The integral reconstructor of $\dot{y}$ is defined to be:

$$
\hat{\dot{y}}=\int_{0}^{t} u(\tau) d \tau
$$

The relation between the structural estimate of $\dot{y}$ of the velocity and the actual value of the velocity state is given by,

$$
\hat{\dot{y}}=\dot{y}-\dot{y}(0)-\xi t
$$

The presence of an unstable ramp error between the integral reconstructor of the velocity and the actual velocity value, prompts us to use a complementary double integral compensating control action on the basis of the position tracking error. We have the following result:

Proposition 1. Given the perturbed dynamical system, described in (7), the following dynamical feedback control law

$$
\begin{aligned}
u= & \ddot{y}^{*}-k_{3}\left(\hat{\dot{y}}-\dot{y}^{*}\right)-k_{2} e_{y}(t)-k_{1} \int_{0}^{t} e_{y}(\tau) d \tau \\
& -k_{0} \int_{0}^{t} \int_{0}^{\tau} e_{y}(\sigma) d \sigma d \tau
\end{aligned}
$$

with $\hat{y}$ defined by (9), forces the output $y$ to asymptotically exponentially track the desired reference trajectory, $y^{*}(t)$.

Proof. Substituting equation (11) into equation (7), yields the following closed loop tracking error dynamics:

$$
\begin{aligned}
& \ddot{e_{y}}+k_{3}\left(\hat{y}-\dot{y}^{*}\right)+k_{2} e_{y}+k_{1} \int_{0}^{t} e_{y}(\tau) d \tau \\
& +k_{0} \int_{0}^{t} \int_{0}^{\tau} e_{y}(\sigma) d \sigma d \tau=0
\end{aligned}
$$

Using (10) one obtains,

$$
\begin{aligned}
& \ddot{e}_{y}+k_{3} \dot{e}_{y}+k_{2} e_{y}+k_{1} \int_{0}^{t} e_{y}(\tau) d \tau \\
& +k_{0} \int_{0}^{t} \int_{0}^{\tau} e_{y}(\sigma) d \sigma d \tau=k_{3}(\dot{y}(0)+\xi t)
\end{aligned}
$$

Taking two time derivatives in (13) the introduced disturbance due to the integral reconstructor is annihilated as follows:

$$
e_{y}^{(4)}+k_{3} e_{y}^{(3)}+k_{2} \ddot{e}_{y}+k_{1} \dot{e}_{y}+k_{0} e_{y}=0
$$


The justification of this last step is readily obtained by defining the following state variables along with their initial conditions,

$$
\begin{aligned}
\rho_{1} & =\int_{0}^{t} e_{y}(\tau) d \tau-\left(k_{3} / k_{1}\right) y(0), \\
\rho_{1}(0) & =-\left(k_{3} / k_{1}\right) y(0) \\
\rho_{2} & =\int_{0}^{t} \int_{0}^{\tau} e_{y}(\lambda) d \lambda d \tau-\left(k_{3} / k_{0}\right) \xi t, \\
\rho_{2}(0) & =0 \\
\rho_{3} & =\dot{\rho}_{2}=\int_{0}^{t} e_{y}(\tau) d \tau-\left(k_{3} / k_{0}\right) \xi \\
\rho_{3}(0) & =-\left(k_{3} / k_{0}\right) \xi
\end{aligned}
$$

The closed loop system reads then as follows,

$$
\frac{d}{d t} \chi=A \chi
$$

with $\chi=\left(e_{y}, \dot{e}_{y}, \rho_{1}, \rho_{2}, \rho_{3}\right)^{T}$ and

$$
A=\left[\begin{array}{cccc}
0 & 1 & 0 & 0 \\
-k_{2} & -k_{3} & -k_{1} & -k_{0} \\
1 & 0 & 0 & 0 \\
0 & 0 & 0 & 1 \\
1 & 0 & 0 & 0
\end{array}\right]
$$

The characteristic polynomial associated with the matrix $A$ is readily found to be given by

$$
P_{A}(s)=s^{4}+k_{3} s^{3}+k_{2} s^{2}+k_{1} s+k_{0}
$$

Finally, by choosing $k_{3}, k_{2}, k_{1}, k_{0}$ such that the polynomial (15) has all its roots located on the left half of the complex plane, $\mathbb{C}$, the tracking error, $e_{y}$, decreases exponentially asymptotically to zero as a function of time.

Remark 2. Notice that the GPI controller (11) can also be written as a classic compensation network (expressed in the frequency domain). From (11) and (9),

$$
\begin{aligned}
u(t) & =u^{*}(t)-k_{3} \int_{0}^{t} e_{u}(\tau) d \tau-k_{2} e_{y}(t)-k_{1} \int_{0}^{t} e_{y}(\tau) d \tau \\
& -k_{0} \int_{0}^{t} \int_{0}^{\tau} e_{y}(\sigma) d \sigma d \tau
\end{aligned}
$$

Using the fact that, $e_{u}=u-u^{*}(t)$, and applying the Laplace transform to the last expression, we have,

$$
e_{u}(s)=-k_{3} \frac{e_{u}(s)}{s}-k_{2} e_{y}(s)-k_{1} \frac{e_{y}(s)}{s}-k_{0} \frac{e_{y}(s)}{s^{2}}
$$

Re-ordering the last equation we have:

$$
e_{u}(s)=-\left[\frac{k_{2} s^{2}+k_{1} s+k_{0}}{s\left(s+k_{3}\right)}\right] e_{y}(s)
$$


In other words

$$
u(s)=s^{2} y^{*}(s)-\left[\frac{k_{2} s^{2}+k_{1} s+k_{0}}{s\left(s+k_{3}\right)}\right] e_{y}(s)
$$

\subsection{Generalized proportional integral observers}

Consider the following $n$-th order scalar nonlinear differential equation,

$$
y^{(n)}=\phi\left(t, y, \dot{y}, \ddot{y}, \cdots, y^{(n-1)}\right)+k u
$$

where $\phi$ is a smooth nonlinear scalar function, $k \in \mathbb{R}$ and $u$ is a control input. We state the following definitions and assumptions:

Definition 3. Define the following time function:

$$
\varphi: t \mapsto \phi\left(t, y(t), \dot{y}(t), \ddot{y}(t), \cdots, y^{(n-1)}(t)\right)
$$

i.e., denote by $\varphi(t)$, the value of $\phi$ for a certain solution $y(t)$ of (20) for a fixed set of $\square$ nite initial conditions. In other words; $\varphi(t)=\phi\left(t, y(t), \dot{y}(t), \ddot{y}(t), \cdots, y^{(n-1)}(t)\right)$, where $y(t)$ is a smooth bounded solution of $E q$. (20) from a certain set of $\square$ nite initial conditions.

\section{Assumptions 4.}

- We assume that a unique, smooth, bounded solution, $y(t)$, exists for the nonlinear differential equation, (20), for every given set of $\square$ nite initial conditions.

- The values of the function, $\varphi(t)$, are unknown, except for the fact that they are known to be uniformly, absolutely, bounded for every smooth bounded function, $y(t)$, which is a solution of Eq. (20).

- For any positive integer $p$, we can find a small positive, real number, $\delta_{p}$, such that $\varphi^{(p)}(t)$ is uniformly absolutely bounded, i.e.,

$$
\sup _{t \geq 0}\left|\varphi^{(p)}(t)\right|<\delta_{p}, \forall p \in \mathbb{Z}^{+}<\infty
$$

- The following system

$$
y^{(n)}=\varphi(t)+k u
$$

with $u$ as a known system input, and $\varphi(t)$ unknown but bounded with negligible high order derivatives after some integer order $p$ is assumed to capture, from a signal processing viewopoint, all the essential features of the nonlinear system (20).

\subsubsection{A GPI observer approach to state estimation of unknown dynamics}

We formulate the state estimation problem for the system (20) via GPI observers as follows: Under the above assumptions, given the noise-free measurement of $y(t), u(t)$, it is desired to estimate the natural state variables (or: phase variables) of the system (20), given by $y(t), \dot{y}(t)$, $\ddot{y}(t), \ldots, y^{(n-1)}(t)$, via the use of the natural equivalence of system (20) with the simpli ed uncertain system given by (23).

The solution to the simultaneous state and perturbation estimation problem can be achieved via the use of an extended version of the traditional linear Luenberger observer, that we address here as GPI observer, as follows. 
Proposition 5. Luviano-Juárez et al. (2010) Under the assumptions given above. For a system of the form (23), the following observer

$$
\begin{aligned}
\dot{\hat{y}}_{1} & =\lambda_{p+n-1}\left(y-\hat{y}_{1}\right)+\hat{y}_{2} \\
\dot{\hat{y}}_{2} & =\lambda_{p+n-2}\left(y-\hat{y}_{1}\right)+\hat{y}_{3} \\
\vdots & \\
\dot{\hat{y}}_{n} & =\lambda_{p}\left(y-\hat{y}_{1}\right)+k u+\rho_{1} \\
\dot{\rho}_{1} & =\lambda_{p-1}\left(y-\hat{y}_{1}\right)+\rho_{2} \\
\dot{\rho}_{2} & =\lambda_{p-2}\left(y-\hat{y}_{1}\right)+\rho_{3} \\
\vdots & \\
\dot{\rho}_{p-2} & =\lambda_{2}\left(y-\hat{y}_{1}\right)+\rho_{p-1} \\
\dot{\rho}_{p-1} & =\lambda_{1}\left(y-\hat{y}_{1}\right)+\rho_{p} \\
\dot{\rho}_{p} & =\lambda_{0}\left(y-\hat{y}_{1}\right) \\
\hat{y}_{i} & =\hat{y}^{(i-1)}
\end{aligned}
$$

asymptotically exponentially reconstructs, via the observer variables: $\hat{y}_{1}, \hat{y}_{2}, \ldots, \hat{y}_{n}$, the phase variables $y, \dot{y}, \cdots, y^{(n-1)}, y^{(n)}$, while the observer variables $\rho_{1}, \rho_{2}, \ldots$, respectively, reconstruct in an asymptotically exponentially fashion, the perturbation input $\varphi(t)$ and its time derivatives $\dot{\varphi}(t), \ldots$ modulo a small error, uniformly bounding the reconstruction error $\varepsilon=y-\hat{y}(t)=y-\hat{y}_{1}$, and its first $n-1$ - th order time derivatives provided the design parameters, $\lambda_{0}, \cdots, \lambda_{p+n-1}$ are chosen so that the roots of the associated polynomial in the complex variable s:

$$
P(s)=s^{p+n}+\lambda_{p+n-1} s^{p+n-1}+\lambda_{p+n-2} s^{p+n-2}+\ldots+\lambda_{1} s+\lambda_{0}
$$

are all located deep in the left half of the complex plane.

Proof. Define, as suggested in the Proposition, the estimation error as follows:

$$
\varepsilon(t) \triangleq y(t)-\widehat{y}_{1}(t)
$$

taking $p+n$ time derivatives in last equation, and using the reconstruction error dynamics for $\varepsilon$, derivable from the observer equations, leads to the following perturbed reconstruction error dynamics:

$$
\varepsilon^{(p+n)}+\lambda_{p+n-1} \varepsilon^{(p+n-1)}+\lambda_{p+n-2} \varepsilon^{(p+n-2)}+\cdots+\lambda_{1} \dot{\varepsilon}+\lambda_{0} \varepsilon=\varphi^{(p)}(t)
$$

which is a perturbed $n+p$ - th order linear time invariant system, whose perturbation input is given by $\varphi^{(p)}(t)$. Given that the characteristic polynomial $P(s)$, corresponding to the unperturbed output reconstruction error system, has its roots in the left half of the complex plane, then the Bounded Input Bounded Output (BIBO) stability condition is assured, Kailath (1979) since, uniformly in $t,\left|\varphi^{(p)}(t)\right|<\gamma_{p}$. Thus, the output reconstruction error, $\varepsilon$, and its first $n+p-1$ time derivatives are ultimately constrained to a disk in the reconstruction error phase space of arbitrary small radius which is further decreased as the roots of the dominating characteristic polynomial are chosen farther and farther into the left half of the complex plane. 


\section{Problem formulation}

Consider the following dynamic model describing the two-phase equivalent model of a three-phase motor controlled by the phase voltages $u_{S a}$ and $u_{S b}$ with state variables given by: $\theta$, describing the rotor angular position, $\omega$ being the rotor angular velocity, $\psi_{R a}$ and $\psi_{R b}$, representing the unmeasured rotor fluxes, while $i_{S a}$ and $i_{S b}$ are taken to be the stator currents.

$$
\begin{aligned}
\frac{d \theta}{d t} & =\omega \\
\frac{d \omega}{d t} & =\mu\left(i_{S b} \psi_{R a}-i_{S a} \psi_{R b}\right)-\frac{\tau_{L}}{J} \\
\frac{d \psi_{R a}}{d t} & =-\eta \psi_{R a}-n_{p} \omega \psi_{R b}+\eta M i_{S a} \\
\frac{d \psi_{R b}}{d t} & =-\eta \psi_{R b}+n_{p} \omega \psi_{R a}+\eta M i_{S b} \\
\frac{d i_{S a}}{d t} & =\eta \beta \psi_{R a}+\beta n_{p} \omega \psi_{R b}-\gamma i_{S a}+\frac{u_{S a}}{\sigma L_{S}} \\
\frac{d i_{S b}}{d t} & =\eta \beta \psi_{R b}-\beta n_{p} \omega \psi_{R a}-\gamma i_{S b}+\frac{u_{S b}}{\sigma L_{S}}
\end{aligned}
$$

with

$$
\begin{gathered}
\eta:=\frac{R_{R}}{L_{R}}, \beta:=\frac{M}{\sigma L_{R} L_{S}}, \mu:=\frac{n_{p} M}{J L_{R}}, \\
\gamma:=\frac{M^{2} R_{R}}{\sigma L_{R}^{2} L_{S}}+\frac{R_{S}}{\sigma L_{S}}, \sigma:=1-\frac{M^{2}}{L_{R} L_{S}}
\end{gathered}
$$

$R_{R}$ and $R_{S}$ are, respectively, the rotor and stator resistances, $L_{R}$ and $L_{S}$ represent, respectively, the rotor and stator inductances, $M$ is mutual inductance constant, $J$ is the moment of inertia and $n_{p}$ is the number of pole pairs. The signal $\tau_{L}$ is the unknown load torque perturbation input. We adopt the complex notation like in Sira-Ramirez, Beltran-Carbajal \& Blanco-Ortega (2008). Define the following complex variables:

$$
\begin{aligned}
& \psi_{R}=\psi_{R a}+j \psi_{R b}=\left|\psi_{R}\right| e^{j \theta_{\psi}} \\
& u_{S}=u_{S a}+j u_{S b}=\left|u_{S}\right| e^{j \theta_{u}} \\
& i_{S}=i_{S a}+j i_{S b}=\left|i_{S}\right| e^{j \theta_{i}}
\end{aligned}
$$

The induction motor dynamics is rewritten as

$$
\begin{aligned}
\frac{d^{2} \theta}{d t^{2}} & =\mu \mathcal{I} m\left(\bar{\psi}_{R} i_{S}\right)-\tau_{L}(t) \\
\frac{d\left|\psi_{R}\right|^{2}}{d t} & =-2 \eta\left|\psi_{R}\right|^{2}+2 \eta M \mathcal{R} e\left(\bar{\psi}_{R} i_{S}\right) \\
\frac{d \theta_{\psi}}{d t} & =n_{p} \omega+\frac{R_{r} M}{L_{r}\left|\psi_{R}\right|^{2}} \mathcal{I} m\left(\bar{\psi}_{R} i_{S}\right) \\
\frac{d i_{S}}{d t} & =\beta\left(\eta+n_{p} \omega\right) \psi_{R}-\gamma i_{S}+\frac{1}{\sigma L_{S}} u
\end{aligned}
$$


where $\bar{\psi}_{R}$ denotes the complex conjugate of the complex rotor flux $\psi_{R}$.

We have, thus, established explicit, separate, dynamics for the squared rotor flux magnitude and for the rotor flux phase angle. This representation, clearly exhibits a decoupling property of the model which allows one to, independently, control the square of the flux magnitude and the angular position by means of the stator currents acting as auxiliary control input variables. This representation also establishes that the complex flux phase angle is largely determined by the manner in which the angular position is controlled by the stator currents.

The problem formulation is as follows: Given the induction motor dynamics, given a desired constant reference level for the rotor flux magnitude $\left|\psi_{R}^{*}\right|>0$, and given a smooth reference trajectory $\theta^{*}(t)$ for the angular position of the motor shaft, the control problem consists in finding a feedback control law for the phase voltages $u_{S a}$ and $u_{S b}$ in such a way that $\theta$ is forced to track the given reference trajectory, $\theta^{*}$, while the rotor flux magnitude stabilizes around the desired value, $\left|\psi_{R}^{*}\right|$. Such objectives are to be achieved in spite of the presence of unknown but bounded perturbation inputs represented by 1) the load torque, $\tau_{L}(t)$, in the rotor shaft dynamics and 2) the effects of motor nonlinearities acting on the current dynamics through possibly unknown parameters.

\section{Control strategy}

The GPI observer-controller design considerations will be based on the following simplified, linear, models lumping the external load disturbances and the system nonlinearities in the form of components of an unknown perturbation input vector, as follows:

$$
\begin{aligned}
\frac{d^{2} \theta}{d t^{2}} & =\mu \mathcal{I} m\left(\bar{\psi}_{R} i_{S}\right)+\xi_{1}(t) \\
\frac{d\left|\psi_{R}\right|^{2}}{d t} & =-2 \eta\left|\psi_{R}\right|^{2}+2 \eta M \mathcal{R} e\left(\bar{\psi}_{R} i_{S}\right) \\
\frac{d \theta_{\psi}}{d t} & =n_{p} \omega+\frac{R_{r} M}{L_{r}\left|\psi_{R}\right|^{2}} \mathcal{I} m\left(\bar{\psi}_{R} i_{S}\right) \\
\frac{d i_{S}}{d t} & =\frac{1}{\sigma L_{S}} u_{S}+\xi(t)
\end{aligned}
$$

where $\xi_{1}(t)=-\tau_{L}(t), \xi(t)=\xi_{2}(t)+j \xi_{3}(t)$ are considered as disturbance inputs, with $\xi_{1}(t)$ representing the unknown load perturbation input, and $\xi(t)$ represents nonlinear and linear additive dissipation terms, depending on the stator currents $i_{S a}, i_{S b}$ and the angular velocity. The currents $i_{S a}$ and $i_{S b}$ can be directly measured; on the other hand, rotor fluxes must be estimated. For the flux estimation, we used a real time simulation of the rotor flux equation dynamics. Parameters $\eta, n_{p}, M$ need to be known; on the other hand, the lumped parameter $\mu$ must be estimated. Nevertheless, in our control scheme, such a task is not entirely necessary due to the remarkable robustness of the scheme and a reasonable guess can be used in the controller expression for such parameters. The disturbance functions $\xi_{1}(t), \xi(t)$ can be envisioned to contain the rest of the system dynamics, including some un-modeled dynamics (which can be of a rather complex nonlinear character). In these terms, we also lump disturbances of additive nature such as frictions and the effects generated by parameter variations during the system operation and even the effects of inaccurate parameter estimations. These perturbation inputs, however, do not contain any control terms.

For the correct tracking of angular position, it is necessary to provide additional control loops for other variables. As it is customary, the flux modulus has to be regulated to a certain value 
in order to assure the efficient operation of the induction machine avoiding possible saturation effects.

The proposed control scheme consists in a two stage feedback controller design. The first stage controls the angular position of the motor shaft to track the reference signal $\theta^{*}(t)$ by means of the stator currents taken as auxiliary control inputs. As a collateral objective it is desired to have the flux magnitude converging towards a given constant value $\left|\psi_{R}^{*}\right|^{1}$. For this stage, the control strategy is implemented by means of a GPI based observer controller, Cortés-Romero et al. (2009). As a result of the first stage a set of desirable current trajectories is synthesized. The obtained currents are thus taken as output references for the second multi-variable stage. The second stage designs a discontinuous feedback controller to force the actual currents to track the obtained current references in the first stage. In the second stage the stator voltages are the control inputs. The following section deals with the flux reconstructor.

\subsection{Flux reconstruction}

Note that the complex rotor flux $\psi_{R}$ satisfies the following dynamics:

$$
\frac{d \psi_{R}}{d t}=-\left(\eta+j n_{p} \omega\right) \psi_{R}+\eta M i_{S}
$$

A simple reconstruction dynamics, with self stable reconstruction error dynamics, is given by

$$
\frac{d \widehat{\psi}_{R}}{d t}=-\left(\eta+j n_{p} \omega\right) \widehat{\psi}_{R}+\eta M i_{S}
$$

The complex reconstruction error $e=\psi_{R}-\widehat{\psi}_{R}$ satisfies then the linear dynamics:

$$
\frac{d e}{d t}=-\left(\eta+j n_{p} \omega\right) e
$$

whose unique eigenvalue has a strictly negative real part (and a time varying complex part). Thus the complex error, $e$, satisfies $e \rightarrow 0$ in an exponentially asymptotic manner. Thus, henceforth, when we use $\psi$ in the expressions it is implicitly assumed that it is obtained from the proposed reconstructor undergoing the exponential convergence process $\widehat{\psi} \rightarrow \psi$.

\subsection{Outer loop controller design stage}

For this first design stage we consider the following dynamics:

$$
\begin{aligned}
\frac{d^{2} \theta}{d t^{2}} & =\mu \mathcal{I} m\left(\bar{\psi}_{R} i_{S}\right)+\xi_{1}(t) \\
\frac{d\left|\psi_{R}\right|^{2}}{d t} & =-2 \eta\left|\psi_{R}\right|^{2}+2 \eta M \mathcal{R} e\left(\bar{\psi}_{R} i_{S}\right) \\
\frac{d \theta_{\psi}}{d t} & =n_{p} \omega+\frac{R_{r} M}{L_{r}\left|\psi_{R}\right|^{2}} \mathcal{I} m\left(\bar{\psi}_{R} i_{S}\right)
\end{aligned}
$$

with the complex stator current $i_{S}$ acting as auxiliary control input.

We propose the following complex controller:

\footnotetext{
${ }^{1}$ Inaccurate parameters may cause minimal variations in the flux regulation, however, the angular position remains unaffected due to the robustness of the controller.
} 


$$
i_{S}=\frac{\psi_{R}}{\left|\psi_{R}\right|^{2}}\left[\frac{\left|\psi_{R}^{*}\right|^{2}}{M}+j v\right]
$$

with $\left|\psi_{R}^{*}\right|$ being the desired flux magnitude reference value and $v$ is an auxiliary control input. In closed loop, the squared modulus of the rotor flux satisfies

$$
\frac{d\left|\psi_{R}\right|^{2}}{d t}=-2 \eta\left[\left|\psi_{R}\right|^{2}-\left|\psi_{R}^{*}\right|^{2}\right]
$$

then, $\left|\psi_{R}\right| \rightarrow\left|\psi_{R}^{*}\right|=$ constant, in an exponential asymptotic manner.

On the other hand, the angular position dynamics satisfies, in closed loop, the perturbed dynamics.

$$
\frac{d^{2} \theta}{d t^{2}}=\mu v+\xi_{1}(t)
$$

with $\xi_{1}(t)$ assumed to be time-varying, unknown but bounded signal, directly related to the load torque, and $v$ being a control input yet to be specified. The specification of the auxiliary control input $v$ is made on the basis of a GPI controller. With an abuse in the notation, we use the following GPI observer based controller:

$$
v=\frac{1}{\mu}\left[\ddot{\theta}^{*}-\frac{k_{1 \theta} s+k_{0 \theta}}{s+k_{2 \theta}}\left(\theta-\theta^{*}\right)-\hat{\xi}_{1}\right]
$$

where $\hat{\xi}_{1}$ is the on-line estimate of the unknown signal $\xi_{1}(t)$. For the estimation of the disturbance function $\xi_{1}(t)$, we assume that $\xi_{1}(t)$ is bounded with bounded low order time derivatives and negligible higher order time derivatives. Such signals may be locally approximated in a self-updated manner, thanks to the internal model principle, by a generic representative of a family of time polynomial signals, of fixed finite, relatively low degree, and free coefficients. Thus, modeling $\xi_{1}(t)$ by means of, say, a 5th degree family of polynomials, the following GPI observer, containing a suitable internal model of the perturbation input, is proposed:

$$
\begin{aligned}
& \frac{d \hat{\theta}}{d t}=\lambda_{7}(\theta-\hat{\theta})+\hat{\theta} \\
& \frac{d \hat{\theta}}{d t}=\lambda_{6}(\theta-\hat{\theta})+\mu v+\rho_{1 \theta} \\
& \dot{\rho}_{1 \theta}=\lambda_{5}(\theta-\hat{\theta})+\rho_{2 \theta} \\
& \dot{\rho}_{2 \theta}=\lambda_{4}(\theta-\hat{\theta})+\rho_{3 \theta} \\
& \dot{\rho}_{3 \theta}=\lambda_{3}(\theta-\hat{\theta})+\rho_{4 \theta} \\
& \dot{\rho}_{4 \theta}=\lambda_{2}(\theta-\hat{\theta})+\rho_{5 \theta} \\
& \dot{\rho}_{5 \theta}=\lambda_{1}(\theta-\hat{\theta})+\rho_{6 \theta} \\
& \dot{\rho}_{6 \theta}=\lambda_{0}(\theta-\hat{\theta}) \\
& \hat{\xi}_{1}=\rho_{1 \theta}
\end{aligned}
$$

The estimation error is defined as $e_{\theta}:=\theta-\hat{\theta}$ satisfies the following injected dynamics:

$$
\begin{aligned}
& e_{\theta}^{(8)}+\lambda_{7} e_{\theta}^{(7)}+\lambda_{6} e_{\theta}^{(6)}+\lambda_{5} e_{\theta}^{(5)}+\lambda_{4} e_{\theta}^{(4)} \\
& +\lambda_{3} e_{\theta}^{(3)}+\lambda_{2} \ddot{e}_{\theta}+\lambda_{1} \dot{e}_{1}+\lambda_{0} e_{\theta}=\xi_{1}^{(6)}(t)
\end{aligned}
$$


By an appropriate choice of the coefficients, $\lambda_{i} ; i=0,1, \ldots, 7$ the characteristic polynomial in the complex variable $s$

$$
\begin{aligned}
p_{\hat{\xi}_{1}}(s)= & s^{8}+\lambda_{7} s^{7}+\lambda_{6} s^{6}+\lambda_{5} s^{5}+\lambda_{4} s^{4} \\
& +\lambda_{3} s^{3}+\lambda_{2} s^{2}+\lambda_{1} s+\lambda_{0}
\end{aligned}
$$

can be made into a Hurwitz polynomial. The estimation error is assured to be ultimately bounded by a small disk around the origin in the estimating error state space which can be further reduced by adjusting the observer gains to produce eigenvalues sufficiently far at the left half of the complex plane. Under these circumstances, $\hat{\theta} \rightarrow \theta$ and $\hat{\theta} \rightarrow \dot{\theta}$ modulo an arbitrarily small error and, subsequently, it is clear that $\rho_{1 \theta} \rightarrow \xi_{1}$ with the same convergence rate (See Sira-Ramírez et al., Feliu-Battle, Sira-Ramirez, Feliu-Batlle, Beltran-Carbajal \& Blanco-Ortega (2008)).

The closed loop characteristic polynomial for the angular position tracking error response is just:

$$
p_{\theta}(s)=s^{3}+k_{2 \theta} s^{2}+k_{1 \theta} s+k_{0 \theta}
$$

while the characteristic polynomial governing the exponential convergence of the squared norm of the flux towards the desired constant value, is given by

$$
p_{\left|\psi_{R}\right|^{2}}(s)=s+2 \eta
$$

\subsection{Inner loop controller design stage}

Consider now the simplified perturbed stator currents dynamics (30):

$$
\frac{d i_{S}}{d t}=\frac{1}{\sigma L_{S}} u_{S a v}+\xi(t)
$$

We regard this simplified dynamics as an average perturbed representation of the stator current dynamics which is to be regulated by means of a switched input voltages strategy, similar in nature to those arising from the variable structure, sliding mode controller, approach ( the reader is referred to, Castillo-Toledo et al. (2008); Utkin et al. (1999) and references therein).

Denote the three phase currents, and three phase voltages, respectively, by $i_{1}, i_{2}, i_{3}$, and $u_{1}, u_{2}$, $u_{3}$. Define the stator current vector as an $\mathbb{R}^{2}$ vector with components, $i_{S a}, i_{S b}$, while the stator voltage vector, is also defined as a vector with components: $u_{S a}, u_{S b}$, with the perturbation vector also defined as: $\xi=\left[\begin{array}{ll}\xi_{2} & \xi_{3}\end{array}\right]^{T}$. These quantities are transformed from the phase current vector, $i$, the phase voltage vector, $u$, and the phase sliding surface, $\sigma$, as follows:

$$
\begin{aligned}
& i=\left[\begin{array}{l}
i_{1} \\
i_{2} \\
i_{3}
\end{array}\right]=\sqrt{\frac{3}{2}}\left[\begin{array}{cc}
2 / 3 & 0 \\
-1 / 3 & 1 / \sqrt{3} \\
-1 / 3 & -1 / \sqrt{3}
\end{array}\right]\left[\begin{array}{l}
i_{S a} \\
i_{S b}
\end{array}\right]=P\left[\begin{array}{l}
i_{S a} \\
i_{S b}
\end{array}\right] \\
& u=\left[\begin{array}{l}
u_{1} \\
u_{2} \\
u_{3}
\end{array}\right]=\sqrt{\frac{3}{2}}\left[\begin{array}{cc}
2 / 3 & 0 \\
-1 / 3 & 1 / \sqrt{3} \\
-1 / 3 & -1 / \sqrt{3}
\end{array}\right]\left[\begin{array}{l}
u_{S a} \\
u_{S b}
\end{array}\right]=P\left[\begin{array}{l}
u_{S a} \\
u_{S b}
\end{array}\right] \\
& \bar{\xi}=\sqrt{\frac{3}{2}}\left[\begin{array}{cc}
2 / 3 & 0 \\
-1 / 3 & 1 / \sqrt{3} \\
-1 / 3 & -1 / \sqrt{3}
\end{array}\right]\left[\begin{array}{l}
\xi_{2} \\
\xi_{3}
\end{array}\right]=P\left[\begin{array}{l}
\xi_{2} \\
\xi_{3}
\end{array}\right]
\end{aligned}
$$


Let us define the following vector sliding surface in operational calculus terms:

$$
\begin{aligned}
& \bar{\sigma}=\left[\begin{array}{lll}
\sigma_{1} & \sigma_{2} & \sigma_{3}
\end{array}\right]^{T}=-\left(\frac{s+z}{s}\right) e_{i} \\
& e_{i}=i-i^{*}(t)
\end{aligned}
$$

and the switched control input is given by

$$
u=W \operatorname{sign}(\bar{\sigma})=W\left[\begin{array}{l}
\operatorname{sign}\left(\sigma_{1}\right) \\
\operatorname{sign}\left(\sigma_{2}\right) \\
\operatorname{sign}\left(\sigma_{3}\right)
\end{array}\right]
$$

Proceeding as in the introductory example, let us consider the following Lyapunov candidate function

$$
V=\frac{1}{2} \bar{\sigma}^{T} \bar{\sigma}
$$

We have:

$$
\begin{aligned}
& \dot{V}=\bar{\sigma}^{T} \dot{\bar{\sigma}}=-\bar{\sigma}^{T} \dot{e}_{i}-z \bar{\sigma}^{T} e_{i} \\
& \dot{e}_{i}=\frac{W}{\sigma L_{s}} \operatorname{sign}(\bar{\sigma})+\bar{\xi}-\frac{d i^{*}}{d t}
\end{aligned}
$$

We have:

$$
\begin{aligned}
\dot{V} & =-\bar{\sigma}^{T} \dot{e}_{i}-z \bar{\sigma}^{T} e_{i}= \\
& =-\frac{W}{\sigma L_{s}}\left(\left|\sigma_{1}\right|+\left|\sigma_{2}\right|+\left|\sigma_{3}\right|\right)-\bar{\sigma}^{T} \bar{\xi}+\bar{\sigma}^{T} \frac{d i^{*}}{d t}-z \bar{\sigma}^{T} e_{i}
\end{aligned}
$$

Thus, for a large enough voltages amplitude $W$, the sliding condition $\bar{\sigma}^{T} \dot{\bar{\sigma}}<0$ is satisfied and the vector of phase sliding coordinates converges towards $\sigma_{1}=0, \sigma_{2}=0, \sigma_{3}=0$, in, a finite time under the switching control $u$.

The closed loop behavior under sliding mode condition can be obtained using the equivalent control method. Using the invariance conditions $\bar{\sigma}=\dot{\bar{\sigma}}=0$, we have:

$$
\dot{e}_{i}+z e_{i}=0
$$

Therefore, the tracking error converges to zero asymptotically. A schematic diagram of the control methodology is given in figure 2 . 


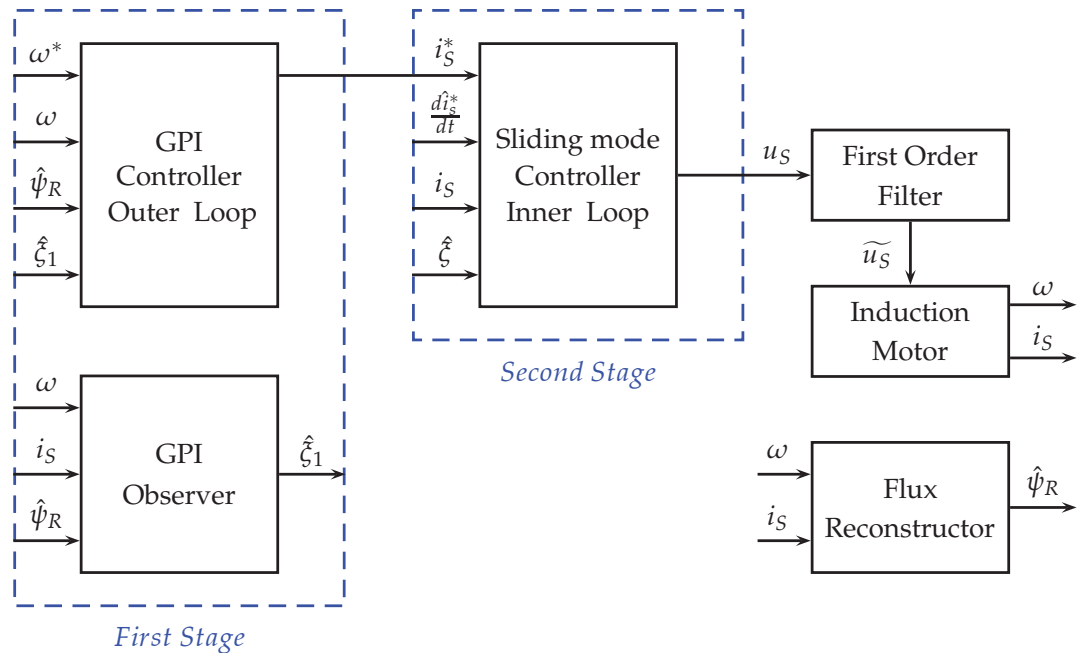

Fig. 2. Control schematics.

\section{Experimental results}

We illustrate the proposed control approach by some experiments on an actual induction motor test bed. The experimental induction motor prototype includes the following parameters: $J=4.5 \times 10^{-4}\left[\mathrm{Kg} \mathrm{m}^{2}\right], n_{p}=1, M=0.2768[\mathrm{H}], L_{R}=0.2919[\mathrm{H}], L_{S}=0.2919$ $[\mathrm{H}], R_{S}=5.12[\Omega], R_{R}=2.23[\Omega]$. The flux absolute desired value was $0.5872[\mathrm{~Wb}]$.

The sliding mode surface parameter was $z=350$. The output of the sliding control was filtered by means of a first order low pass filter of Bessel type with cut frequency of 750 $\mathrm{rad} / \mathrm{s}$. The angle measurement was obtained using an incremental encoder with $10000 \mathrm{PPR}$. The desired closed loop tracking error was set in terms of the characteristic polynomial $P_{\theta}(s)=\left(s^{2}+2 \zeta \omega_{n}+\omega_{n}^{2}\right)(s+p)$, with $\zeta=1, \omega_{n}=330, p=320$, and the observer injection error characteristic polynomial was $P_{\hat{\xi}}=\left(s^{2}+2 \zeta_{1} \omega_{n 1}+\omega_{n 1}^{2}\right)^{4}$, with $\zeta_{1}=2, \omega_{n 1}=27$.

The controller was devised in a MATLAB - xPC Target environment using a sampling period of .1 [ms]. The communication between the plant and the controller was performed by two data acquisition devises. The analog data acquisition was performed by a National Instruments PCI-6025E data acquisition card, and the digital outputs as well as the encoder reading for the position sensor were performed in a National Instruments PCI-6602 data acquisition card. The voltage and current signals are conditioned for adquisition system by means of low pass filters with cut frequency of $1[\mathrm{kHz}]$. The interconnection of the modules can be appreciated in a block diagram form as depicted in figure 3.

The output reference trajectory to be tracked, was set to be a biased sinusoidal wave of the form:

$$
\theta^{*}=\left\{\begin{array}{cc}
0 & 0 \leq t<2 \\
1+\sin (t-\pi / 2) & 2 \leq t \leq 10
\end{array}\right.
$$

Figure 4 shows an accurate position tracking with respect to the desired trajectory. As we can see in figure 5, the control loops indirectly regulate the flux magnitude whose error is under $5 \times 10^{-3}[\mathrm{~Wb}]$. The sliding mode control induces a slight high frequency wave envelope. However, as depicted in figure 6, the average current tracks perfectly the desired reference 
currents in both phases (a and b). Notice that the control voltages (figure 7) are continuous, but they remain affected by the discontinuities despite the filtering effect.

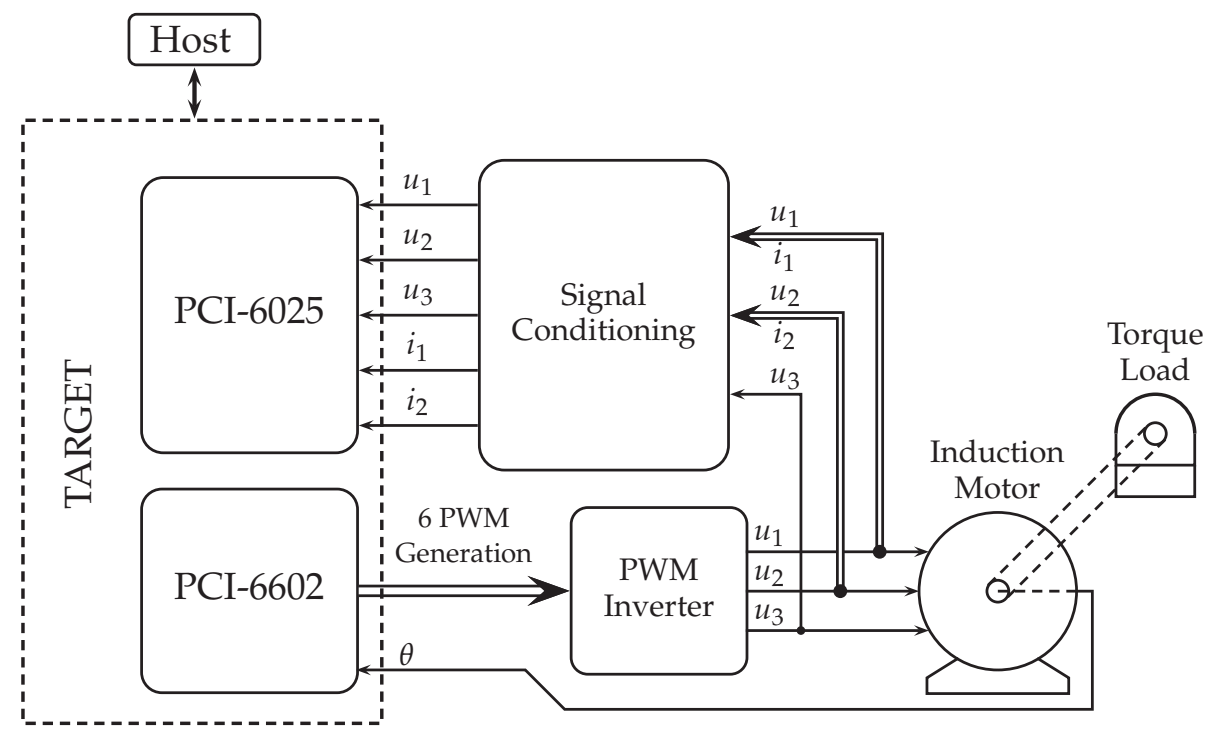

Fig. 3. Block diagram of the control system
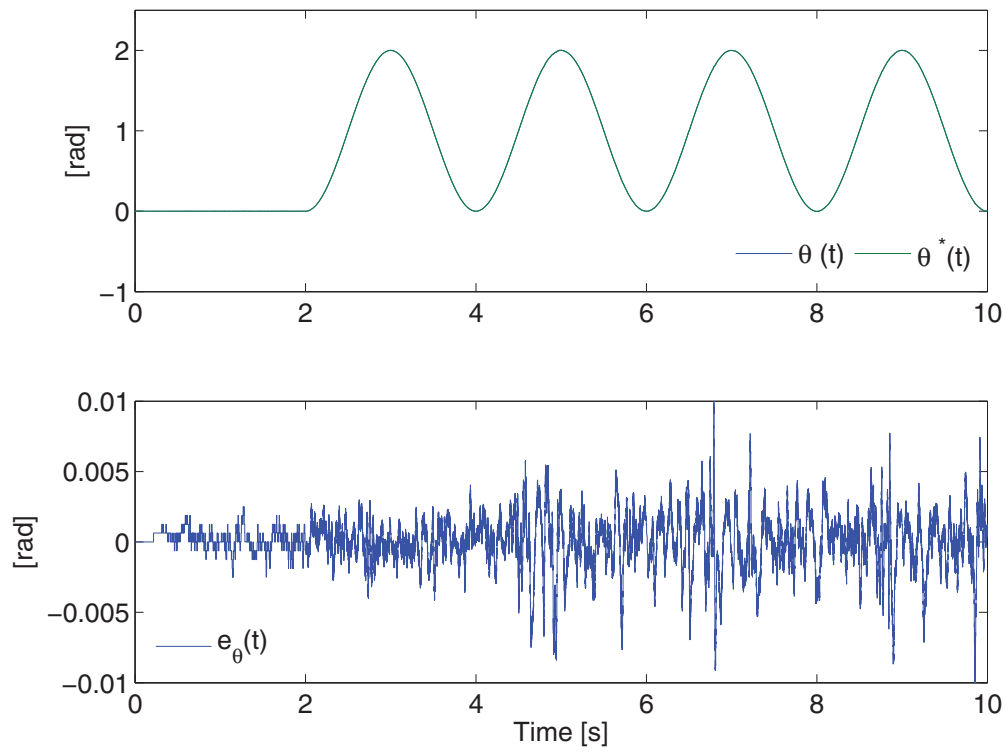

Fig. 4. Position trajectory tracking. 
Finally, to illustrate the robustness of the strategy, we applied a load torque with a voltage controlled brake. The applied voltage was implemented by a variable resistor array, where its value was randomly adjusted by a manual tuning. The disturbance estimation, as well as the load torque observation and the auxiliary input $v$ can be seen in figure 8 .
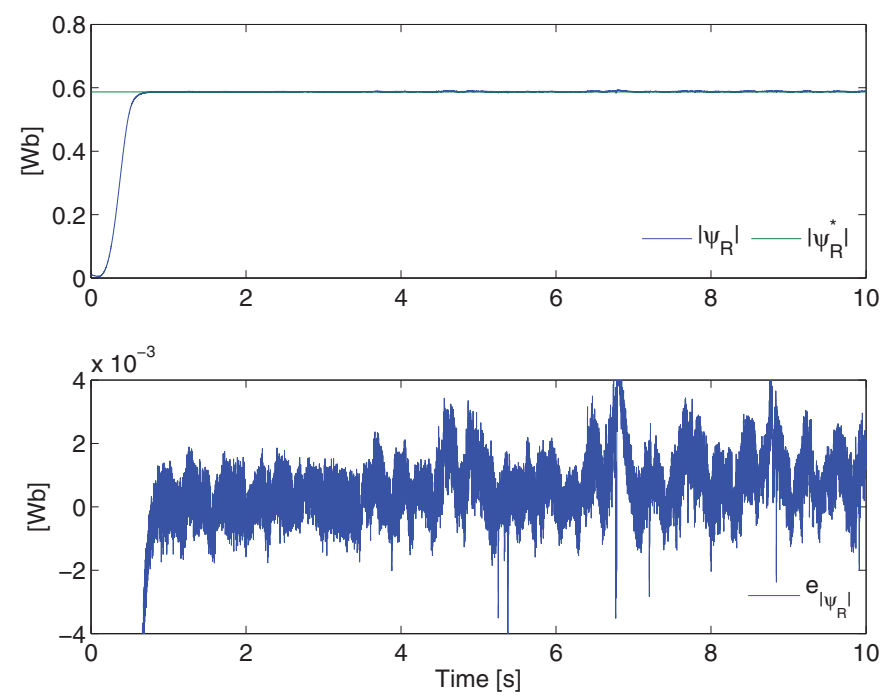

Fig. 5. Flux magnitude regulation.
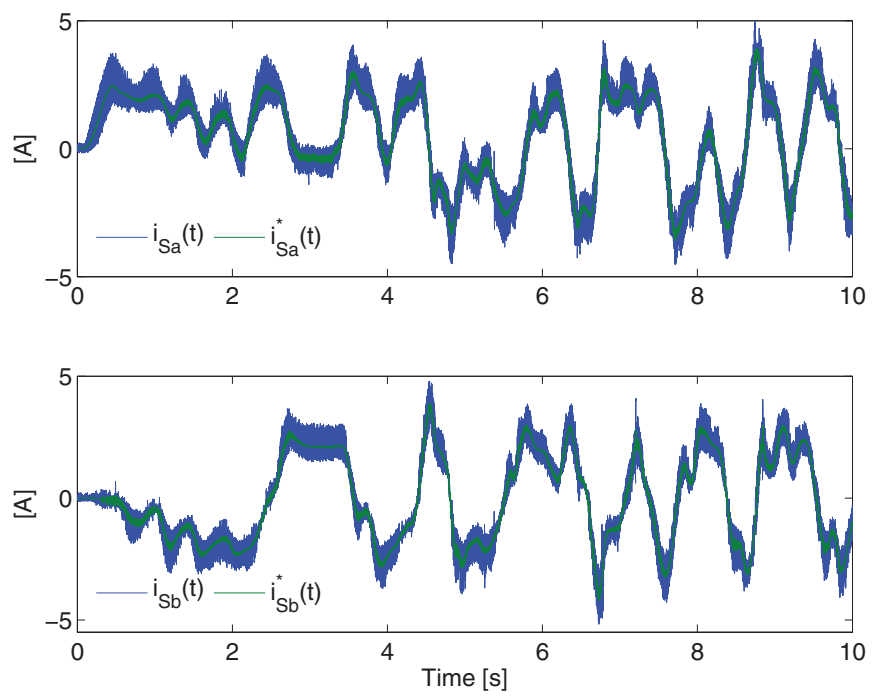

Fig. 6. Current tracking. 

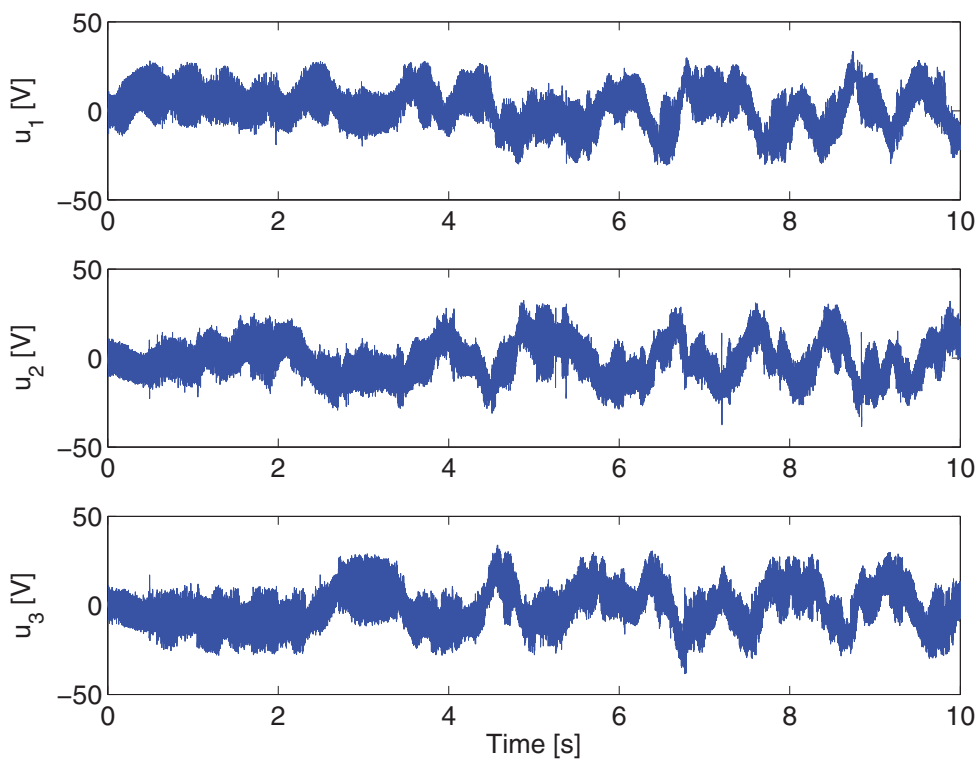

Fig. 7. Stator voltages.
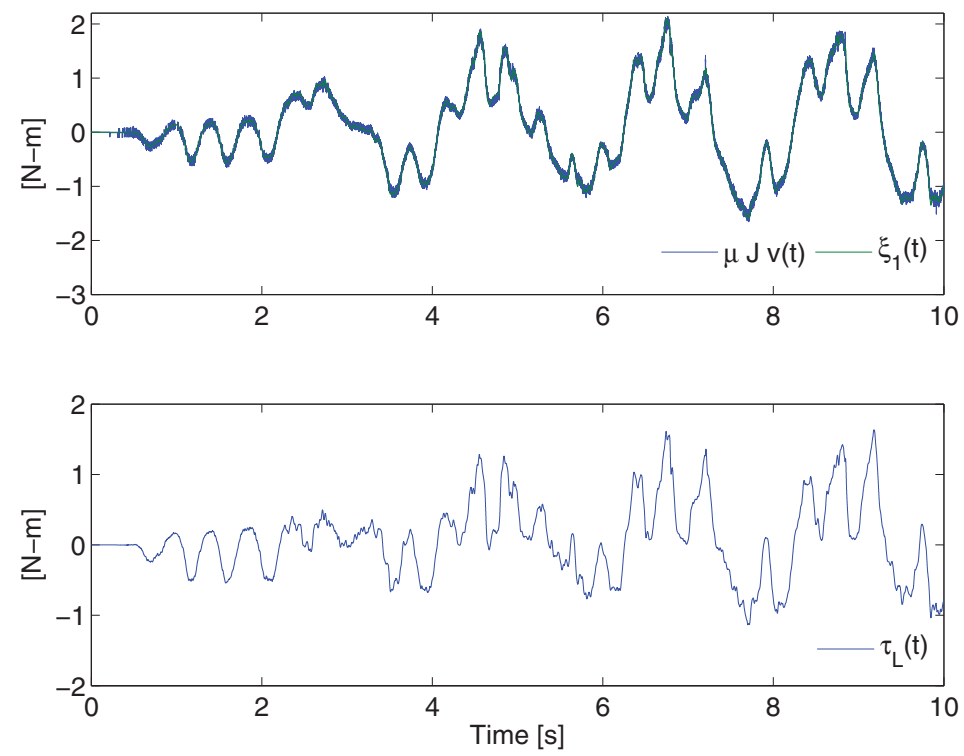

Fig. 8. Load torque, disturbance estimation and auxiliary control input $v$. 


\section{Concluding remarks}

In this work, a combination of two control loops, one discontinous sliding mode control and another based on the combination of GPI control and GPI disturbance observer was proven to be quite suitable for robust position control and tracking tasks in an induction motor system. An experimental test was carried out where the plant is subject to unforseen external disturbances and un-modeled nonlinear state dependent perturbations. Here, we used the disturbance estimates to carry out the disturbance rejection and for canceling the effects of un-modeled disturbance inputs in the motor, in the case of the mechanical subsystem.

Since the strategy regulates the flux, as a collateral task, since the current variables are well regulated, then the experimental flux variable showed accurate results.

The behavior of the proposed scheme is based upon the correct setting of the characteristic polynomial of the observer which guarantees the correct cancelation of disturbance terms by means of its estimation process.

\section{References}

Castillo-Toledo, B., Gennaro, S. D., Loukianov, A. \& Rivera, J. (2008). Hybrid control of induction motors via sampled closed representations, IEEE Transactions on Industrial Electronics 55(10): 3758-3771.

Chiasson, J. (2005). Modeling and High-Performance Control of Electric Machines, New York: Wiley.

Cortés-Romero, J., Luviano-Juárez, A. \& Sira-Ramírez, H. (2009). Robust GPI controller for trajectory tracking for induction motors, Proceedings of the 2009 IEEE International Conference on Mechatronics, Málaga, Spain.

Fliess, M., Levine, J., Martin, P. \& Rouchon, P. (1995). Flatness and defect of non-linear systems: introductory theory and examples, International Journal of Control 61(6): 1327-1361.

Fliess, M., Marquez, R., Delaleau, E. \& Sira-Ramírez, H. (2002). Correcteurs proportionnels integraux généralisés, ESAIM: Control, Optimisation and Calculus of Variations 7: 23-41. URL: $h$ ttp://dx.doi.org/doi/10.1051/cocv:2002002

Fliess, M., Marquez, R. \& Mounier, H. (2002). An extension of predictive control, pid regulators and smith predictors to some linear delay systems, International Journal of Control 75: 728 Ü743.

Fliess, M. \& Sira-Ramírez, H. (2004). Reconstructeurs d'etat, C.R. Acad. Sci. Paris 338(1): 91-96. t. 332, Série I.

Harnefors, L. \& Hinkkanen, M. (2008). Complete stability of reduced-order and full-order observers for sensorless IM drives, IEEE Transactions on Industrial Electronics 55(3): $1319-1329$.

Hasan, S. \& Husain, I. (2009). A Luenberger-Sliding Mode Observer for Online Parameter Estimation and Adaptation in High-Performance Induction Motor Drives, IEEE Transactions on Industry Applications 45(2): 772-781.

Hernández, V. M. \& Sira-Ramírez, H. (2003). Generalized p.i. control for global robust position regulation of rigid robot manipulators, 2003 American Control Conference, Denver Colorado, USA.

Kailath, T. (1979). Linear Systems (Prentice-Hall Information and System Science Series), Prentice Hall.

URL: http://www.amazon.ca/exec/obidos/redirect?tag=citeulike09-20Eamp;path=ASIN/0135 369614

Luviano-Juárez, A., Cortés-Romero, J. \& Sira-Ramírez, H. (2010). Synchronization of chaotic oscilators by means of proportional integral observers, International Journal of Bifurcation and Chaos 20(5): 1509ú-1517. 
Melfi, M., Evon, S. \& McElveen, R. (2009). Induction versus permanent magnet motors, IEEE Industry Applications Magazine 15(6): 28 -35.

Sira-Ramírez, H. \& Agrawal, S. (2004). Differentially flat systems, Marcel Dekker Inc.

Sira-Ramirez, H., Beltran-Carbajal, F. \& Blanco-Ortega, A. (2008). A robust generalized proportional integral controller for the induction motor, International Workshop on Variable Structure Systems VSS '08. pp. 89-94.

Sira-Ramirez, H., Feliu-Batlle, V., Beltran-Carbajal, F. \& Blanco-Ortega, A. (2008). Sigma-delta modulation sliding mode observers for linear systems subject to locally unstable inputs, Proceedings of the 16th Mediterranean Conference on Control and Automation, pp. 344-349.

Sira-Ramírez, H. \& Silva-Ortigoza, R. (2006). Control design techniques in power electronics devices, Power systems, Springer-Verlag, London.

Slotine, J. \& Li, W. (1991). Applied Nonlinear Control, Prentice Hall.

Toliyat, H., Levi, E. \& Raina, M. (2003). A review of RFO induction motor parameter estimation techniques, IEEE Transactions on Energy Conversion 18(2): 271-283.

Utkin, V., Guldner, J. \& Shi, J. (1999). Sliding Mode Control Electromechanical System, CRC Press. 


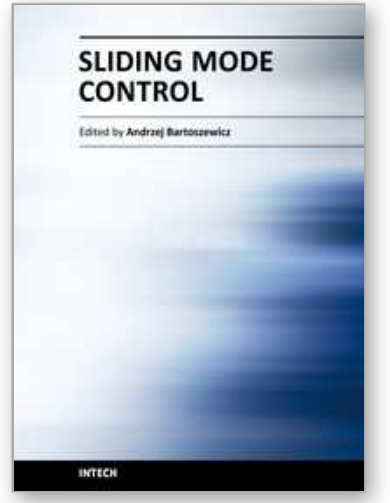

\author{
Sliding Mode Control \\ Edited by Prof. Andrzej Bartoszewicz
}

ISBN 978-953-307-162-6

Hard cover, 544 pages

Publisher InTech

Published online 11, April, 2011

Published in print edition April, 2011

The main objective of this monograph is to present a broad range of well worked out, recent application studies as well as theoretical contributions in the field of sliding mode control system analysis and design. The contributions presented here include new theoretical developments as well as successful applications of variable structure controllers primarily in the field of power electronics, electric drives and motion steering systems. They enrich the current state of the art, and motivate and encourage new ideas and solutions in the sliding mode control area.

\title{
How to reference
}

In order to correctly reference this scholarly work, feel free to copy and paste the following:

John Cortés-Romero, Alberto Luviano-Juárez and Hebertt Sira-Ramírez (2011). Sliding Mode Control Design for Induction Motors: An Input-Output Approach, Sliding Mode Control, Prof. Andrzej Bartoszewicz (Ed.), ISBN: 978-953-307-162-6, InTech, Available from: http://www.intechopen.com/books/sliding-mode-control/slidingmode-control-design-for-induction-motors-an-input-output-approach

\section{INTECH}

open science | open minds

\section{InTech Europe}

University Campus STeP Ri

Slavka Krautzeka 83/A

51000 Rijeka, Croatia

Phone: +385 (51) 770447

Fax: +385 (51) 686166

www.intechopen.com

\section{InTech China}

Unit 405, Office Block, Hotel Equatorial Shanghai

No.65, Yan An Road (West), Shanghai, 200040, China

中国上海市延安西路65号上海国际贵都大饭店办公楼 405 单元

Phone: +86-21-62489820

Fax: +86-21-62489821 
(C) 2011 The Author(s). Licensee IntechOpen. This chapter is distributed under the terms of the Creative Commons Attribution-NonCommercialShareAlike-3.0 License, which permits use, distribution and reproduction for non-commercial purposes, provided the original is properly cited and derivative works building on this content are distributed under the same license. 